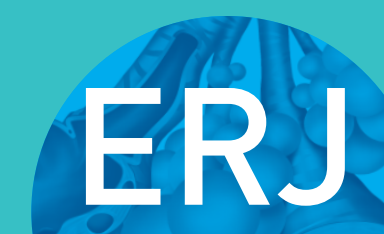

open research
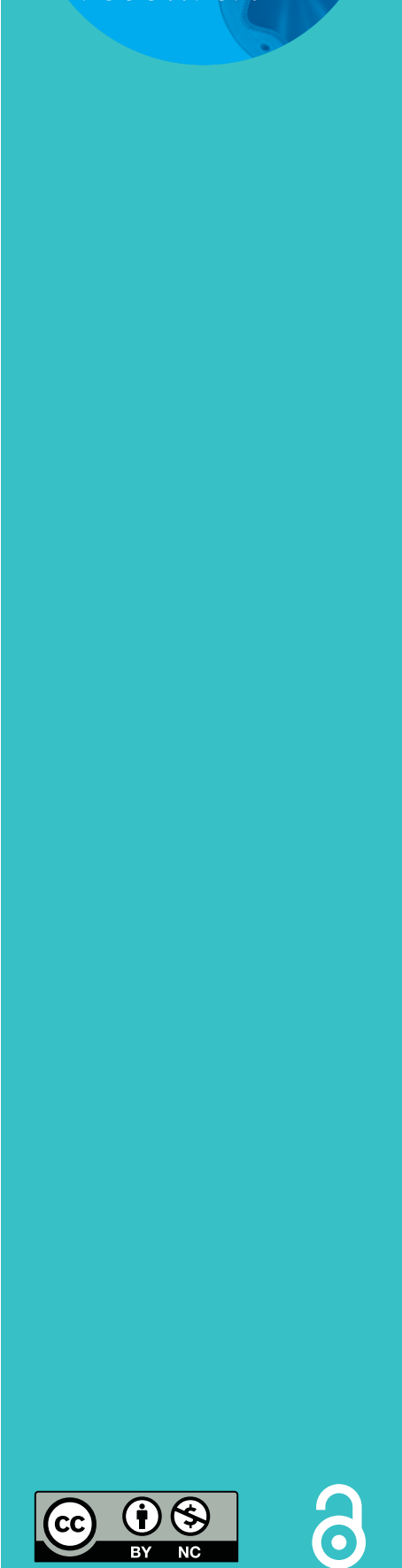

\section{Incidence, comorbidity and survival rate of hypersensitivity pneumonitis: a national population-based study}

\author{
Anne Hald Rittig (i) ${ }^{1}$, Ole Hilberg ${ }^{2}$, Rikke Ibsen $^{3}$ and Anders Løkke
}

Affiliations: ${ }^{1}$ Dept of Respiratory Medicine, Aarhus University Hospital, Aarhus, Denmark. ${ }^{2}$ Dept of Respiratory Medicine, Hospital Vejle, Vejle, Denmark. ${ }^{3}$ i2minds, Aarhus, Denmark.

Correspondence: Anders Løkke, Dept of Respiratory Medicine, Aarhus University Hospital, Nørrebrogade 44, 8000 Aarhus C, Denmark. E-mail: aloekkedgmail.com

\section{ABSTRACT}

Background and objectives: Hypersensitivity pneumonitis (HP) is a rare disease, which can lead to premature death. Few studies have investigated HP on a national level. The objective of this study was to investigate incidence, survival rate, and comorbidity of HP in Denmark.

Methods: Using the Danish National Patient Registry we identified all patients with a first-time diagnosis of HP between 1998 and 2010. Patients with HP were matched 1:4 with controls by sex, age and geography in this case-control study. Comorbidity 3 years prior to diagnosis was explored by the Charlson score index. Survival rates were assessed using Kaplan-Meier curves and hazard ratios.

Results: We identified 753 patients during the observation period equalling an average HP incidence of 1.16 per 100000 citizens. Patients with HP had a significantly higher Charlson score index when compared with the matched controls and an increased risk of dying (hazard ratio 1.98, CI 1.61-2.58, SE $0.14, \mathrm{p}<0.001)$. Survival rates of HP were lower at all time points when compared with the matched control population. The decline in survival was observed for both male and female patients with HP with no clear sex difference. Most deaths were related to diseases of the heart and lungs.

Conclusions: In this Danish longitudinal nationwide observational study we found an increased Charlson score index combined with a higher mortality without sex difference among patients with HP compared with a healthy control group, mainly due to diseases of the heart and lungs.

@ERSpublications

Hypersensitivity pneumonitis is rare and associated with increased mortality rates especially during the first 2 years following diagnosis http://bit.ly/20o7tiR

Cite this article as: Rittig AH, Hilberg O, Ibsen R, et al. Incidence, comorbidity and survival rate of hypersensitivity pneumonitis: a national population-based study. ERJ Open Res 2019; 5: 00259-2018 [https://doi.org/10.1183/23120541.00259-2018].

This article has supplementary material available from openres.ersjournals.com

Received: 27 Dec 2018 | Accepted after revision: 20 July 2019

Copyright $\odot$ ERS 2019. This article is open access and distributed under the terms of the Creative Commons Attribution Non-Commercial Licence 4.0. 


\section{Introduction}

Hypersensitivity pneumonitis (HP) is a rare interstitial lung disease (ILD) characterised by pulmonary inflammation that may lead to fibrosis. The inflammation and fibrosis are caused by an exaggerated immune response due to repeatedly inhaled antigens [1-3]. Thus, the diagnosis of HP includes a history of antigen exposure and relevant pulmonary symptoms. This should lead to further investigations, including pulmonary function testing, radiologic imaging of the thorax, precipitating antibodies for specific antigen exposures, bronchoalveolar lavage and histological samples from the lungs $[4,5]$.

A range of different organic antigens has been associated with the development of HP. The most common origin of the antigens is animals and vegetables, but HP can also be caused by organic chemicals [6, 7]. In disease development, size and solubility are important characteristics of the antigens. Antigens associated with HP are usually aerosolised and only a few micrometres in diameter, which enable them to reach the periphery of the lung and initiate type III and type IV hypersensitivity reactions [3].

Identifying the antigen is of utmost importance, with avoidance of antigen exposure being a prudent part of HP treatment [8]. Overall prognosis associated with HP is favourable when the disease-mediating antigen is eliminated from the patient's surroundings [9]. However, prognosis varies according to age, the number of episodes and the duration of antigen exposure [10, 11]. Continuous exposure or repeated episodes have been shown to accelerate the age-related proportional decrease in forced expiratory volume in $1 \mathrm{~s}$ and forced vital capacity. Ultimately, HP can result in low diffusing capacity, fibrosis and premature death $[9,12-14]$.

The prevalence of HP differs greatly among, and even within, countries due to factors such as distribution of antigens, culture, geography, seasons and climate [15]. A European study has reported that HP accounts for $4-13 \%$ of all ILD cases [16]. In accordance with these findings, a Danish study has shown that HP accounted for $7 \%$ of all ILD cases [17].

The incidence of HP is believed to be underestimated because of subclinical cases, misdiagnosis and unrecognised disease. Few studies have made epidemiological examinations of incidence and mortality rate $[14,18]$. Therefore, we aimed to evaluate age at diagnosis, incidence, hospital contacts, comorbidity and mortality rate of HP nationwide in Denmark.

\section{Methods}

We conducted a register-based case-control study using the National Patient Registry (NPR) in order to identify all patients diagnosed with HP within the time period 1998 to 2010. The NPR contains time-based information regarding all inpatient and outpatient contacts and diagnoses. International classification systems are used in the NPR including the International Statistical Classification of Diseases and Related Health Problems 10th Revision (ICD-10 Version: 2010). The ICD-10 was introduced in Denmark in 1994 and was used throughout the study period. All Danish patients are registered in the NPR on first-time primary or secondary diagnosis and therefore evaluated in this dataset. The patients were identified by extracting the following first-time primary or secondary diagnosis from the NPR: "J67 Hypersensitivity pneumonitis due to organic dust", "J67.0 Farmer lung", "J67.1 Bagassosis", "J67.2 Bird fancier lung", "J67.3 Suberosis", "J67.4 Maltworker lung”, “J67.5 Mushroom-worker lung”, "J67.6 Maple-bark-stripper lung", "J67.7 Air-conditioner and humidifier lung”, "J67.8 Hypersensitivity pneumonitis due to other organic dusts" and "J67.9 Hypersensitivity pneumonitis due to unspecified organic dust". Our analysis enabled us to select all patients diagnosed with HP in the NPR, but did not allow us to distinguish between acute, subacute and chronic forms.

Age, sex, marital status and geography-matched controls without HP were randomly selected from the Civil Registration System. Control subjects were matched from the same county (Denmark was subdivided into 16 counties) at the time of diagnosis. Overall, four control subjects were matched for each patient within the same county with an average of 20000 possible controls in each county. More than $99 \%$ of the observations were successfully matched.

The study population was followed through the entire time period or until they died. If a patient was given a HP diagnosis during the first year (1998) we were able to follow that individual 12 years forward in time and observe what happened after diagnosis; while diagnosis of HP in the last year of the observation period (2010) made it possible for us to follow that individual 12 years back in time and register what had happened before diagnosis. If an individual was diagnosed with HP in the middle of the observation period (e.g. 2005) we recorded information both before and after diagnosis (from 1998 to 2010).

The Charlson comorbidity index is a validated tool that stratifies comorbid disease status and is a strong estimator of mortality [19]. The Charlson score index was calculated to evaluate a priori disease status. 
Statistical analysis was done using SAS version 9.1.3. Kaplan-Meier survival distribution curves were estimated stratified on case and control groups as well as age and sex. The Kaplan-Meier survival curves are nonparametric and take into account the censoring of the data. Thus, these curves depict the fraction of patients living for a certain amount of time after diagnosis. A Cox proportional regression model was used to estimate the hazard ratio. Survival models relate the time that passes, before some event occurs. We present data comparing patients with HP with controls. A Cox proportional regression hazard ratio $>1$ indicates a higher mortality rate among patients with HP compared with controls and vice versa. Causes of death were obtained from the Danish Death Registry. This registry uses specific ICD-10 diagnoses, which are classified in larger main groups with other related ICD-10 diagnoses. This study was approved by the Danish Data Protection Agency. Ethical approval was not required as data were anonymous and did not contain any attributable patient data.

\section{Results}

Using the NPR, we identified 753 patients diagnosed with HP and also included 3012 matched controls. Age and sex distribution is displayed in table 1. More men (56.7\%) than women (43.3\%) were diagnosed with HP in Denmark during the period from 1998 to 2010. The percentage of married or cohabiting patients was $65.9 \%$ versus $66.1 \%$ in the control group (table 1 ).

The incidence of HP in Denmark during the study period is shown in table 2. Incidence is presented per 100000 citizens and ranges from 0.89 in 2004 to 1.53 in 2005 with an average incidence of 1.16 per 100000 citizens.

Patients diagnosed with HP had a significant higher risk of dying (hazard ratio 1.98, CI 1.61-2.58, SE 0.14, $\mathrm{p}<0.001$ ) compared with matched controls (table 3). The survival among patients was lower compared with controls from the beginning of the observation period and also at every timepoint throughout the observation period (figure 1).

The decline in survival was observed for both male and female patients with HP with no clear sex difference (figure 2 and table 3). When subdividing into age groups 0-39, 40-49, 50-59, 60-69, 70-79 and $\geqslant 80$ years, a significant reduction in survival was revealed in the age groups $0-39,60-69,70-79$ and $\geqslant 80$ years (table 3 ).

We used the Charlson score index $[19,20] 3$ years prior to diagnosis of HP to compare comorbidities between groups and found a significantly higher Charlson index among patients with HP compared with the control group (table 4). To characterise which diagnoses contributed to the Charlson score, we assembled the most common diagnoses among patients and the control group (table S1). Hospital contacts before HP diagnosis were most often due to unspecific diagnoses or conditions affecting lungs and heart.

The most common causes of death in the patient group are depicted in table 5 together with the corresponding number of deaths in the control group. Most deaths were related to diseases of the heart and lungs.

TABLE 1 Distribution of age, sex and cohabiting status in patients with hypersensitivity pneumonitis (HP) and matched controls

\begin{tabular}{lcc} 
& HP & Controls \\
\hline Male & $427(56.7 \%)$ & $1708(56.7 \%)$ \\
$\begin{array}{l}\text { Female } \\
\text { Age distribution years }\end{array}$ & $326(43.3 \%)$ & $1304(43.3 \%)$ \\
$<20$ & $53(7 \%)$ & \\
$20-29$ & $76(10.1 \%)$ & $212(7 \%)$ \\
$30-39$ & $114(15.1 \%)$ & $304(10.1 \%)$ \\
$40-49$ & $145(19.3 \%)$ & $456(15.1 \%)$ \\
$50-59$ & $155(20.6 \%)$ & $580(19.3 \%)$ \\
$60-69$ & $124(16.5 \%)$ & $620(20.6 \%)$ \\
$70-79$ & $67(8.9 \%)$ & $496(16.5 \%)$ \\
$\geqslant 80$ & $19(2.5 \%)$ & $268(8.9 \%)$ \\
All & $753(100 \%)$ & $76(2.5 \%)$ \\
Married or cohabiting (spouses) & $65.9 \%$ & $3012(100 \%)$ \\
& & $66.1 \%$
\end{tabular}


TABLE 2 Incidence of hypersensitivity pneumonitis (HP) per 100000 citizens

\begin{tabular}{cccc} 
Year & Diagnosed with HP & Population & Incidence per $\mathbf{1 0 0 0 0 0}$ \\
\hline $\mathbf{1 9 9 8}$ & 50 & 5294860 & 0.94 \\
$\mathbf{1 9 9 9}$ & 70 & 5313577 & 1.32 \\
$\mathbf{2 0 0 0}$ & 63 & 5330020 & 1.18 \\
$\mathbf{2 0 0 1}$ & 69 & 5349212 & 1.29 \\
$\mathbf{2 0 0 2}$ & 54 & 5368354 & 1.01 \\
$\mathbf{2 0 0 3}$ & 60 & 5383507 & 1.11 \\
$\mathbf{2 0 0 4}$ & 5397640 & 0.89 \\
$\mathbf{2 0 0 5}$ & 48 & 5411405 & 1.53 \\
$\mathbf{2 0 0 6}$ & 83 & 5427459 & 1.27 \\
$\mathbf{2 0 0 7}$ & 69 & 5447084 & 1.38 \\
$\mathbf{2 0 0 8}$ & 75 & 5475791 & 1.06 \\
\end{tabular}

\section{Discussion}

We conducted a longitudinal Danish national registry study, including age at diagnosis, incidence, comorbidity and the mortality rate of HP. In this study, we showed an average incidence of 1.16 people with HP per 100000 citizens. Furthermore, the patient group had a significantly higher Charlson score. Also, mortality was increased among the patient group compared with the controls, revealing a higher mortality in the age groups $0-39$ and $60-80+$ years.

In Denmark, ICD-10 classification is only used in the secondary healthcare sector, not in the primary healthcare sector (general practitioners). HP is almost exclusively diagnosed, treated and followed in the secondary healthcare sector and therefore we can allow ruling out potential loss of diagnosed cases in the primary health care sector, although misdiagnosis, subclinical as well as unrecognised cases could very well still be an issue. Therefore, the exact incidence of HP is still difficult to estimate with precision. With this in mind, the Danish National Health Service provides free access to healthcare-diminishing diagnostic and referral biases. The data in our study do not allow us to distinguish between acute HP and chronic fibrotic HP in our calculations. Several previous studies have investigated incidences of HP caused by a specific antigen [15], whereas only few other studies have investigated the accumulated incidence of HP [18, 21]. A large, general population study from the UK found an incidence of 0.9 cases per 100000 person-years, which is in accordance with our finding [18]. However, the incidence of HP will vary from country to country along with environmental risk factors, climatic factors and antigens $[15,18]$.

Prevalence of HP among individuals exposed to a certain antigen varies, suggesting that both antigen and individual predisposition influences the expression of clinical disease [22-24]. This might explain why slightly more men than women are diagnosed with HP [18]. However, sex did not affect survival as both men and women diagnosed with HP had a significantly higher mortality compared with the matched controls.

\section{TABLE 3 Mortality among males, females and age groups}

\begin{tabular}{|c|c|c|c|c|c|c|}
\hline & \multicolumn{2}{|c|}{ HP } & \multicolumn{2}{|c|}{ Control } & \multirow[t]{2}{*}{ Hazard ratio $(95 \% \mathrm{CI})$} & \multirow[t]{2}{*}{ p-value } \\
\hline & All & Dead & All & Dead & & \\
\hline Male & 427 & $14.3 \%$ & 1708 & $10.1 \%$ & $1.80(1.32-2.45)$ & $<0.001$ \\
\hline Female & 326 & $7.4 \%$ & 1304 & $4.0 \%$ & $2.62(1.56-4.40)$ & $<0.001$ \\
\hline All & 753 & & 3012 & & $1.98(1.61-2.58)$ & $<0.001$ \\
\hline \multicolumn{7}{|c|}{ Age years } \\
\hline $0-39$ & 243 & $2.9 \%$ & 972 & $0.4 \%$ & $7.00(2.05-23.91)$ & 0.002 \\
\hline $40-49$ & 145 & $2.1 \%$ & 580 & $2.1 \%$ & $1.09(0.30-3.91)$ & 0.894 \\
\hline $50-59$ & 155 & $5.2 \%$ & 620 & $4.8 \%$ & $1.10(0.50-2.40)$ & 0.821 \\
\hline $60-69$ & 124 & $19.4 \%$ & 496 & $13.7 \%$ & $1.75(1.07-2.87)$ & 0.025 \\
\hline $70-79$ & 67 & $46.3 \%$ & 268 & $29.1 \%$ & $2.30(1.45-3.66)$ & $<0.001$ \\
\hline$\geqslant 80$ & 19 & $63.2 \%$ & 76 & $43.4 \%$ & $2.56(1.22-5.37)$ & 0.013 \\
\hline
\end{tabular}


FIGURE 1 Survival curves of patients with hypersensitivity pneumonitis (HP) and matched controls. KaplanMeier curve and survival distribution of patients with HP (blue) and controls (red) estimated using the Cox proportional hazard model.

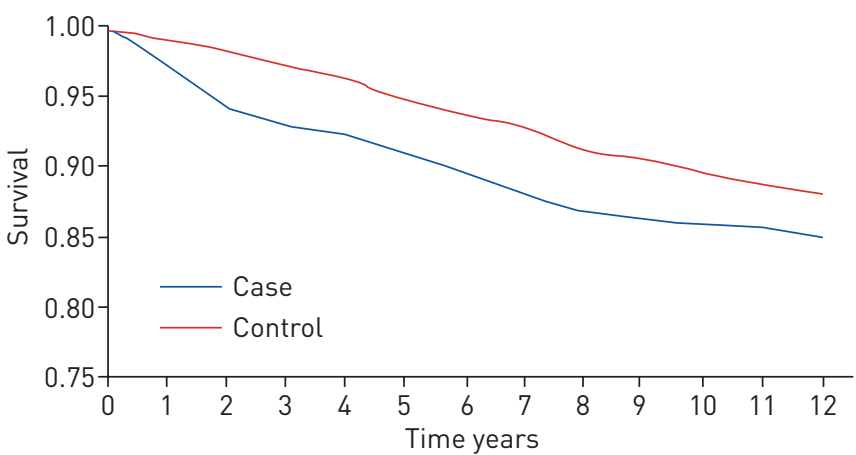

The significant higher Charlson score among the patients with HP may indicate that an a priori risk of other diseases plays a role in the development of HP, speculatively so, perhaps due to an overreacting immune system. We cannot rule out that the increased Charlson score may be due to misdiagnoses (e.g. chronic obstructive pulmonary disease, pneumonia and heart problems) preceding the correct HP diagnosis or side effects from treatments (e.g. corticosteroids). Increased comorbidity among patients with HP would conceivable result in more hospital contacts, which was also the case in this study (table 4 and table S1). More hospital contacts prior to diagnosis of HP may imply an early stage of undiagnosed HP (table S1).

Although evenly distributed throughout the follow-up period, patients with HP aged 0-39 and 60-80+ years had significantly higher mortality compared with healthy controls. Significantly higher mortality in the youngest HP group compared with healthy controls may be due to a low absolute number of deaths among the healthy young ( $0-39$ years) controls. Hence, any covariate that increases death in this age range will be easily noticed. We speculate that the way the immune system works and reacts in in younger individuals may contribute to the higher mortality, but we do not know whether this is the case.

Age has previously been suggested as an important factor in the recovery of lung function [10]. Furthermore, increased mortality among older people diagnosed with HP has been described previously [14] and may be due to an accumulation of comorbidities, making this group more vulnerable. Another possible explanation for the increased mortality rates in both the young and in the elderly could be the treatment with anti-inflammatory drugs (mainly prednisolone), which are often used in high doses [25]. Finally, the increased mortality could be attributed to chronic HP.

A Finnish study found that death occurred more than 5 years after diagnosis in patients diagnosed with farmer's lung [26]. However, most patients with fatal outcome had permanent fibrotic changes on chest radiography at the time of diagnosis, indicative of chronic disease.

The validity of our data depends on the accuracy of ICD-10 coding. Due to limitations of registry studies we have not been able to characterise the people who died. Hence, it is not possible to illuminate the antigen of origin, the degree of lung function or fibrosis in this population. Causes of death have to some

FIGURE 2 Survival curves of patients with hypersensitivity pneumonitis (HP) and matched controls divided by sex. Kaplan-Meier curve and survival distribution of male patients with HP (blue), male controls (red), female HP (green) and female controls (purple) estimated using the Cox proportional hazard model.

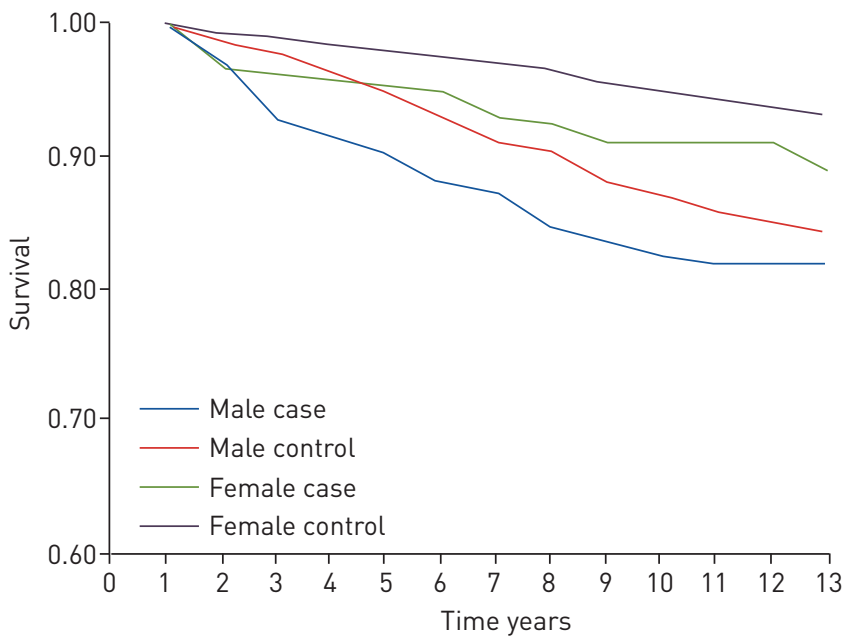


TABLE 4 Charlson score, 3 years prior to diagnosis

\begin{tabular}{lccc} 
& HP & Control & p-value \\
\hline $\begin{array}{lcc}\text { Subjects } \\
\text { Charlson mean } \pm \text { sD }\end{array}$ & 698 & 2792 & \\
\hline HP: hypersensitivity pneumonitis. & $0.1877 \pm 0.5546$ & $0.0852 \pm 0.4146$ & $<0.0001$ \\
\hline
\end{tabular}

\begin{tabular}{|c|c|c|c|c|}
\hline \multirow[t]{2}{*}{ ICD-10 diagnosis } & \multicolumn{2}{|c|}{$\begin{array}{l}\text { Number of } \\
\text { diseased }\end{array}$} & \multicolumn{2}{|c|}{$\begin{array}{l}\text { Percentage of } \\
\text { the population }\end{array}$} \\
\hline & HP & Control & HP & Control \\
\hline Other interstitial pulmonary diseases & 14 & & $1.86 \%$ & \\
\hline Other chronic obstructive pulmonary disease & 12 & 12 & $1.56 \%$ & $0.4 \%$ \\
\hline Chronic ischaemic heart disease or acute myocardial infarction & 6 & 31 & $0.8 \%$ & $1.03 \%$ \\
\hline Malignant neoplasm of bronchus and lung & 5 & 17 & $0.66 \%$ & $0.56 \%$ \\
\hline Malignant neoplasm of rectum & 4 & 7 & $0.53 \%$ & $0.23 \%$ \\
\hline Other ill-defined and unspecified causes of mortality & 4 & 5 & $0.53 \%$ & $0.17 \%$ \\
\hline HP due to organic dust & 4 & & $0.53 \%$ & \\
\hline
\end{tabular}

Left column shows the International Classification of Diseases, 10th Revision (ICD-10) diagnosis/diagnoses cause of death. Columns two and three present the number of diseased in the case versus control group. Columns four and five show the corresponding percentage. HP: hypersensitivity pneumonitis.

degree been clarified in table 5, showing ICD-10-diagnostic cause of death. Due to having few observations it is not possible to do meaningful statistical analysis on the cause of death. Even though HP in general is considered to have a favourable prognosis, we showed an increased mortality combined with causes of death often concerning diseases of the lungs. Even though HP is not registered as the cause of death, the disease may be involved in the development of the other death-causing diseases of the lungs [27, 28].

In conclusion, this Danish nationwide longitudinal retrospective registry study showed an annual average incidence of HP equalling 1.16 per 100000 citizens. Furthermore, we showed a significant higher Charlson score index among patients with HP compared with healthy controls. In accordance with increased comorbidity we found an increased mortality that was evident from the beginning and throughout the entire study period.

Acknowledgements: These data were previously presented as an abstract and poster presentation at the European Respiratory Society 2018 International Congress, Paris, France, 15-19 September, 2018.

Author contributions: A. Løkke is the guarantor of the study. A.H. Rittig, A. Løkke and O. Hilberg conceived the study. A.H. Rittig, A. Løkke and O. Hilberg designed the study. R. Ibsen conducted the data acquisition and statistical analysis of the study. All authors contributed to the interpretation of the findings. A.H. Rittig and A. Løkke prepared the first draft of the manuscript. All authors critically revised and approved the final manuscript.

Conflict of interest: None declared.

\section{References}

1 Reed CE, Barbee RA. Pigeon-breeders' lung: a newly observed interstitial pulmonary disease. JAMA 1965; 193: 261-265.

2 Warren WP, Mandl MA, Rose B. Farmer's lung. Can Med Assoc J 1969; 100: 699-704.

3 Calvert JE, Baldwin CI, Allen A, et al. Pigeon fanciers' lung: a complex disease? Clin Exp Allergy 1999; 29: $166-175$.

4 Sforza GGR, Marinou A. Hypersensitivity pneumonitis: a complex lung disease. Clin Mol Allergy 2017; $15: 6$.

5 Kurup VP, Zacharisen MC, Fink JN. Hypersensitivity pneumonitis. Indian J Chest Dis Allied Sci 2006; 48: $115-128$.

6 Hendrick DJ, Faux JA, Marshall R. Budgerigar-fancier's lung: the commonest variety of allergic alveolitis in Britain. Br Med J 1978; 2: 81-84.

7 Mohr LC. Hypersensitivity pneumonitis. Curr Opin Pulm Med 2004; 10: 401-411.

8 Rose C, King TE Jr. Controversies in hypersensitivity pneumonitis. Am Rev Respir Dis 1992; 145: 1-2. 
9 Fernandez Perez ER, Swigris JJ, Forssen AV, et al. Identifying an inciting antigen is associated with improved survival in patients with chronic hypersensitivity pneumonitis. Chest 2013; 144: 1644-1651.

10 Allen DH, Williams GV, Woolcock AJ. Bird breeder's hypersensitivity pneumonitis: progress studies of lung function after cessation of exposure to the provoking antigen. Am Rev Respir Dis 1976; 114: 555-566.

11 de Gracia J, Morell F, Bofill JM, et al. Time of exposure as a prognostic factor in avian hypersensitivity pneumonitis. Respir Med 1989; 83: 139-143.

12 Schmidt CD, Jensen RL, Christensen LT, et al. Longitudinal pulmonary function changes in pigeon breeders. Chest 1988; 93: 359-363.

13 Vourlekis JS, Schwarz MI, Cherniack RM, et al. The effect of pulmonary fibrosis on survival in patients with hypersensitivity pneumonitis. Am J Med 2004; 116: 662-668.

14 Hanley A, Hubbard RB, Navaratnam V. Mortality trends in asbestosis, extrinsic allergic alveolitis and sarcoidosis in England and Wales. Respir Med 2011; 105: 1373-1379.

15 Terho EO, Heinonen OP, Lammi S, et al. Incidence of clinically confirmed farmer's lung in Finland and its relation to meteorological factors. Eur J Respir Dis Suppl 1987; 152: 47-56.

16 Thomeer MJ, Costabel U, Rizzato G, et al. Comparison of registries of interstitial lung diseases in three European countries. Eur Respir J 2001; 18: Suppl. 32, 114s-118s.

17 Hyldgaard C, Hilberg O, Muller A, et al. A cohort study of interstitial lung diseases in central Denmark. Respir Med 2014; 108: 793-799.

18 Solaymani-Dodaran M, West J, Smith C, et al. Extrinsic allergic alveolitis: incidence and mortality in the general population. QJM 2007; 100: 233-237.

19 Sundararajan V, Henderson T, Perry C, et al. New ICD-10 version of the Charlson comorbidity index predicted in-hospital mortality. J Clin Epidemiol 2004; 57: 1288-1294.

20 Charlson ME, Pompei P, Ales KL, et al. A new method of classifying prognostic comorbidity in longitudinal studies: development and validation. J Chronic Dis 1987; 40: 373-383.

21 Buchvald F, Petersen BL, Damgaard K, et al. Frequency, treatment, and functional outcome in children with hypersensitivity pneumonitis. Pediatr Pulmonol 2011; 46: 1098-1107.

22 Christensen LT, Schmidt CD, Robbins L. Pigeon breeders' disease - a prevalence study and review. Clin Allergy 1975; 5: 417-430.

23 Marx JJ, Guernsey J, Emanuel DA, et al. Cohort studies of immunologic lung disease among Wisconsin dairy farmers. Am J Ind Med 1990; 18: 263-268.

24 Spagnolo P, Richeldi L, du Bois RM. Environmental triggers and susceptibility factors in idiopathic granulomatous diseases. Semin Respir Crit Care Med 2008; 29: 610-619.

25 Idiopathic Pulmonary Fibrosis Clinical Research Network, Raghu G, Anstrom KJ, et al. Prednisone, azathioprine, and N-acetylcysteine for pulmonary fibrosis. N Engl J Med 2012; 366: 1968-1977.

26 Kokkarinen J, Tukiainen H, Terho EO. Mortality due to farmer's lung in Finland. Chest 1994; 106: 509-512.

27 Soumagne T, Chardon ML, Dournes G, et al. Emphysema in active farmer's lung disease. PLoS One 2017; 12: e0178263.

28 Kuramochi J, Inase N, Miyazaki Y, et al. Lung cancer in chronic hypersensitivity pneumonitis. Respiration 2011; 82: $263-267$ 